\title{
Analyse Du Plan National d'Adaptation Aux Changements Climatiques (PNA) Du Burkina Faso Et De Sa Capacité A Atteindre Ses Objectifs
}

\section{Fiacre Basson, Ingénieur Agronome, Environnementaliste et doctorant}

Laboratoire des Matériaux et Environnement (LAME), Université Joseph KI-ZERBO, Ouagadougou, Burkina Faso

Laboratoire d'Etudes et de Recherches sur les Milieux et Territoires (LERMIT), Université Joseph KI-ZERBO, Ouagadougou, Burkina Faso Deutsche Gesellschaft für Internationale Zusammenarbeit (GIZ) GmbH, Ouagadougou, Burkina Faso

François Zougmoré, Professeur Titulaire

Laboratoire des Matériaux et Environnement (LAME), Université Joseph

KI-ZERBO, Ouagadougou, Burkina Faso

Jacques Somda, Docteur en Zoo-économie

Union Internationale de la Conservation de la Nature (UICN), Ouagadougou, Burkina Faso

\section{Jean-Marie Dipama, Professeur Titulaire}

Laboratoire d'Etudes et de Recherches sur les Milieux et Territoires (LERMIT), Université Joseph KI-ZERBO, Ouagadougou, Burkina Faso

Hilaire Wendpagnangdé Ilboudo, Géographe et doctorant Laboratoire des Matériaux et Environnement (LAME), Université Joseph

KI-ZERBO, Ouagadougou, Burkina Faso

\section{Résumé}

Le Burkina Faso ne fait pas exception des impacts négatifs des changements climatiques qui sont de véritables menaces pour le développement durable. Le pays a par conséquent élaboré le Plan National d'Adaptation aux changements climatiques (PNA) pour renforcer sa résilience aux changements climatiques. Pour la réussite de la mise en œuvre du PNA, il est indispensable d'identifier les capacités d'adaptation actuelles de façon approfondie et de fixer des objectifs à moyen terme.

C'est dans cette perspective que s'inscrit la présente étude avec pour objectif d'évaluer les capacités d'adaptation et les objectifs stratégiques du processus du PNA. L'évaluation a été faite par les parties prenantes du PNA 
et ce à l'aide de l'outil SNAP (Stocktaking for National Adaptation Planning) qui dispose de sept facteurs de succès de la planification de l'adaptation aux changements climatiques.

Les résultats montrent que les capacités de suivi-évaluation, de la mise en œuvre et de la participation sont faibles avec des valeurs inférieures à 2 . Les facteurs de succès, (i) l'information climatique, (ii) les capacités humaines, (ii) l'intégration et (iv) la vision et mandat à long terme pour le processus PNA ont été évalués à un niveau moyen (valeurs inférieures à 3 ). Cependant, il est nécessaire d'améliorer considérablement chacun des facteurs de réussite en tant qu'objectif stratégique pour le pays. L'évaluation SNAP a également permis de mettre en évidence des actions et des leçons apprises qui pourront orienter l'opérationnalisation du processus PNA aux niveaux national et sectoriel.

Mots clés : Adaptation Aux Changements Climatiques, Capacités D'adaptation, Changements Climatiques, PNA, SNAP 


\title{
Analysis Of Burkina Faso's National Climate Change Adaptation Plan (NAP) And Its Capacity To Achieve Its Objectives
}

\section{Fiacre Basson, Ingénieur Agronome, Environnementaliste et doctorant}

Laboratoire des Matériaux et Environnement (LAME), Université Joseph KI-ZERBO, Ouagadougou, Burkina Faso

Laboratoire d'Etudes et de Recherches sur les Milieux et Territoires (LERMIT), Université Joseph KI-ZERBO, Ouagadougou, Burkina Faso Deutsche Gesellschaft für Internationale Zusammenarbeit (GIZ) GmbH, Ouagadougou, Burkina Faso

\section{François Zougmoré, Professeur Titulaire}

Laboratoire des Matériaux et Environnement (LAME), Université Joseph

KI-ZERBO, Ouagadougou, Burkina Faso

\section{Jacques Somda, Docteur en Zoo-économie}

Union Internationale de la Conservation de la Nature (UICN), Ouagadougou, Burkina Faso

\section{Jean-Marie Dipama, Professeur Titulaire}

Laboratoire d'Etudes et de Recherches sur les Milieux et Territoires (LERMIT), Université Joseph KI-ZERBO, Ouagadougou, Burkina Faso

Hilaire Wendpagnangdé Ilboudo, Géographe et doctorant Laboratoire des Matériaux et Environnement (LAME), Université Joseph KI-ZERBO, Ouagadougou, Burkina Faso

\begin{abstract}
Burkina Faso is no exception to the negative impacts of climate change, which are real threats to sustainable development. The country has therefore developed the National Adaptation Plan to Climate Change (NAP) to strengthen its resilience to climate change. For the successful implementation of the NAP, it is essential to identify current adaptation capacities in depth and to set medium-term objectives. In this perspective, the objective of this study is to assess the adaptive capacities and the strategic objectives of the NAP process. The assessment was carried out by NAP stakeholders using the SNAP tool (Stocktaking for National Adaptation Planning) which has the seven success factors for climate change adaptation planning.
\end{abstract}


The results show that capacities for monitoring-evaluation, implementation and participation are weak with values below 2. Success factors, (i) climate information, (ii) human capacity, (iii) integration and (iv) long-term vision and mandate for the NAP process were assessed at a medium level (values below 3). However, there is a need to significantly improve each of the success factors as a strategic objective for the country. The SNAP assessment also identified actions and lessons learned that can guide the operationalization of the NAP process at the national and sectoral levels.

Keywords: Climate Change Adaptation, Adaptive Capacity, Climate Change, NAP, SNAP

\section{Introduction}

Les changements climatiques sont une réalité et constituent à long terme des menaces pour le développement des générations actuelles et futures (Adger et al., 2003 ; Barbir et al., 2009). Les impacts des changements climatiques, en raison des gaz à effet de serre déjà présents dans l'atmosphère, se poursuivront pendant au moins les 50 prochaines années (Pielke et al., 2007).

Le développement de mesures d'adaptation semble incontournable pour les Pays les Moins Avancés (PMA) parce qu'ils disposent de faibles capacités adaptatives et que leurs populations sont fortement dépendantes des ressources naturelles sensibles au climat (Niang et al., 2014 ; Millner et Dietz, 2015 ; Gadédjisso-Tossou et al., 2016 ; Ouattara et al., 2019).

La Convention-Cadre des Nations-Unies sur les Changements Climatiques (CCNUCC) a également reconnu que les PMA sont les plus vulnérables aux effets néfastes des changements climatiques et les a accompagnés dans l'élaboration des Programmes d'Action Nationaux d'Adaptation à la vulnérabilité et aux changements climatiques (PANA) (Pramova et al., 2012). Au Burkina Faso, plusieurs auteurs (Traoré et Owiyo, 2013 ; Diarra et al., 2017 ; Dayamba et al., 2019) ont identifié les principaux aléas climatiques telles les variations des régimes pluviométriques et l'augmentation des températures et analysé leurs impacts négatifs sur les moyens de subsistance des populations. Les projections climatiques indiquent une hausse des températures de 2,5 à $5^{\circ} \mathrm{C}$ à 1'horizon 2050 (LAME, 2012). La modélisation des précipitations montre des incertitudes considérables (LAME, 2012 ; Salack et al., 2015 ; Panthou et al., 2018 ; Lejeune et Saeed, 2019), soit à la baisse soit à la hausse. Le pays a élaboré en 2007 son PANA qui visait à mettre en œuvre des actions urgentes de renforcement de la résilience des populations et des écosystèmes aux changements climatiques (UNFCCC, 2010). Mais, sa mise en œuvre a révélé trois difficultés majeures notamment (i) le retard dans son élaboration, (ii) la prise en compte insuffisante des changements 
climatiques dans les politiques et stratégies de développement et (iii) l'insuffisance de financement lors de leur mise en œuvre (MERH, 2015).

Pour résoudre ces difficultés, la CCNUCC a adopté en 2011, le principe de formulation de Plans Nationaux d'Adaptation (PNA) pour les PMA. Ce nouveau cadre de planification contient des informations pratiques pour (i) la réduction de la vulnérabilité des systèmes naturels, sociaux et économiques aux changements climatiques et (ii) l'intégration de l'adaptation aux changements climatiques (ACC) dans les politiques et stratégies de développement actuelles ou à venir. Ainsi en 2015, le Burkina Faso a adopté son PNA (MERH, 2015). S'il est admis que la planification joue un rôle important dans l'adaptation en raison de sa nature prospective et de sa base institutionnelle (Hurlimann et March, 2012), il est alors nécessaire de disposer d'une capacité suffisante des planificateurs et des institutions gouvernementales pour réaliser les objectifs planifiés. Très peu de recherches ont investigué la disponibilité de telles capacités dans les institutions nationales des PMA en général et du Burkina Faso en particulier. Les quelques évaluations des capacités de planification de l'adaptation se sont focalisées sur les communautés locales (Somda et al., 2014). Pourtant, la capacité d'adaptation inclut la capacité des acteurs, des individus, des groupes et des institutions disponibles dans un pays pour mettre en œuvre des mesures d'adaptation efficaces (Chapan et al., 2009 ; GIEC, 2014 ; N'drin et al., 2019). L'objectif global de la présente étude est d'évaluer les capacités de planification de l'ACC au Burkina Faso. De façon spécifique, il s'est agi (i) d'analyser le niveau actuel des facteurs de succès du PNA, (ii) de définir le niveau d'amélioration réaliste de ces facteurs de réussite et (iii) de formuler des actions d'amélioration du niveau actuel des capacités de planification de l'adaptation.

\section{Méthodologie}

\subsection{Zone d'étude}

L'étude a été menée au Burkina Faso qui est situé au cœur de 1'Afrique de l'Ouest avec une superficie de $274000 \mathrm{~km}^{2}$ (Figure 1). Le Burkina Faso a une population de 19 millions d'habitants avec un taux de croissance démographique annuel de $3 \%$ en 2016 (INSD, 2017). Il se caractérise par un climat tropical avec deux saisons très distinctes, la saison des pluies qui va de juin à octobre et la saison sèche de novembre à mai (Ibrahim et al., 2014). Selon la Banque Mondiale (2017), le pays est réparti entre trois zones climatiques notamment i) la zone sahélienne, ii) la zone soudano-sahélienne et iii) la zone soudanienne (Figure 2).

Le Burkina Faso subit déjà les conséquences négatives des changements climatiques. A ce titre, il a élaboré son PNA qui énonce trois tendances principales (i) les températures augmentent plus que les moyennes 
mondiales, (ii) les changements imprévisibles dans la répartition des précipitations et (iii) l'augmentation des phénomènes climatiques extrêmes, tels que la sécheresse et les inondations.

Le PNA propose des mesures d'adaptation à court, moyen et long termes pour sept (07) secteurs prioritaires (agriculture, productions animales, environnement et ressources naturelles, énergie, santé, infrastructures et habitat). A cela s'ajoute, les domaines transversaux (associations féminines et organisations de la société civile. La coordination du PNA est assurée par le Secrétariat Permanent du Conseil National pour le Développement Durable (SP-CNDD) qui est logé au sein du Ministère de l'Environnement, de l'Économie Verte et du Changement Climatique (MEEVCC). En sus du SPCNDD, d'autres acteurs institutionnels jouent un rôle prépondérant dans le processus du PNA. Ces principales structures sont les secteurs ministériels couverts par le PNA, la société civile, les organisations scientifiques et les partenaires techniques et financiers.

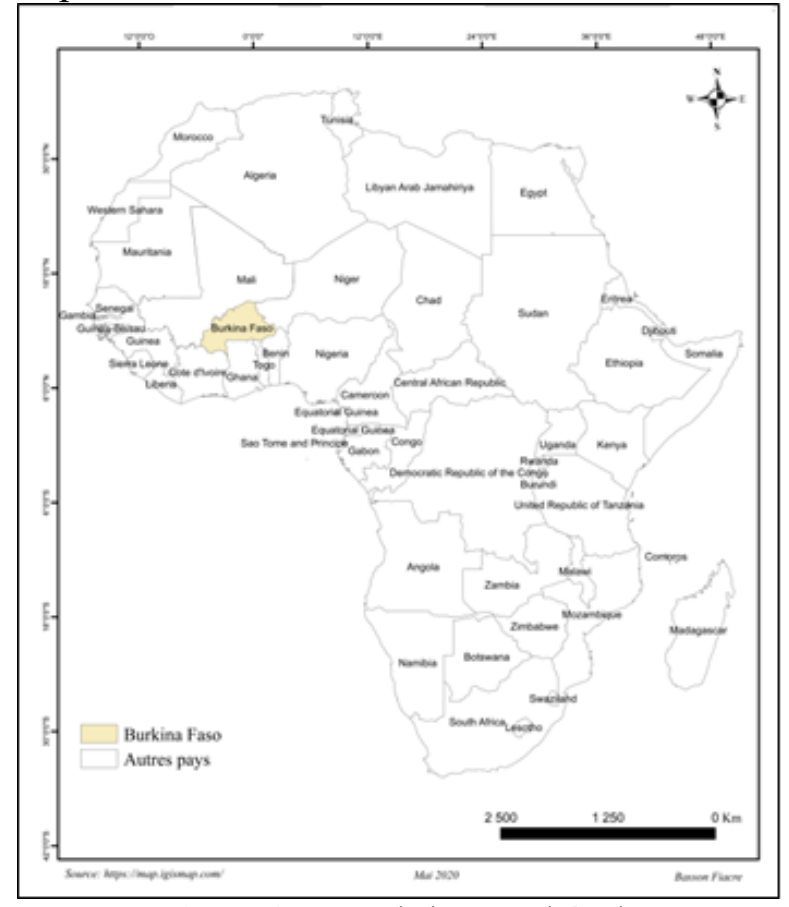

Figure 1. Carte de la zone d'étude 


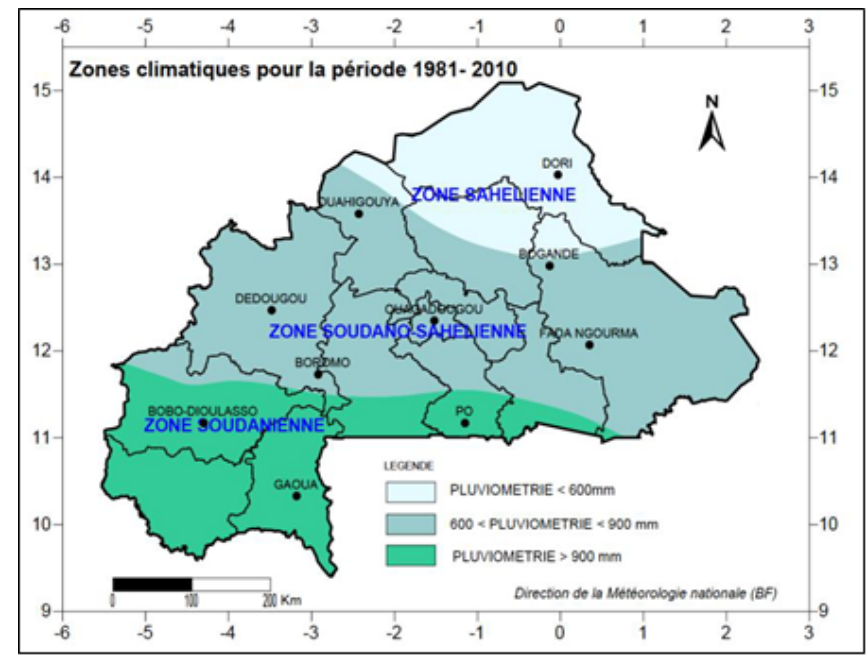

Figure 2. Zones climatiques du Burkina Faso

\subsection{Collecte des données}

\subsubsection{L'outil SNAP}

Pour l'évaluation des capacités de l'ACC, plusieurs auteurs (Somda et al., 2014 ; Karani et Kariuki, 2017 ; GIZ, 2017, 2018) ont utilisé entre autres (i) les ateliers des parties prenantes et/ou d'experts, (ii) les méthodes combinées (études documentaires et des entrevues) et (iii) les enquêtes avec questionnaire. Dans cette étude, l'outil SNAP a été utilisé pour faire l'état des lieux des capacités nationales pour la planification de l'ACC (GIZ, 2014). Il a été choisi pour sa forte valeur heuristique, sa facilité d'utilisation et son application antérieure dans de nombreux pays (Albanie, Bénin, Togo, Tunisie, Mauritanie, Sénégal et Thailande). Le SNAP permet de faire le bilan des capacités de planification actuelles et souhaitées afin d'élaborer et mettre en œuvre un PNA. Il est fondé sur sept (07) facteurs de succès qui ont été identifiés par la GIZ sur la base des Directives techniques sur la planification de l'adaptation élaborées par le Groupe d'experts des PMA de la CCNUCC. Ces facteurs de réussite sont pertinents pour la planification des politiques climatiques dans les économies en développement et émergentes ainsi que des pays industrialisés. Ils se présentent comme suit :

(i) l'information climatique traduit la disponibilité et la qualité des projections climatiques, des études de vulnérabilité et des options techniques d'adaptation ;

(ii) les capacités humaine et institutionnelle correspondent aux capacités des parties prenantes et des institutions à coordonner les processus d'adaptation et à exploiter et gérer les informations climatiques ;

(iii) la vision à long terme et le mandat évoquent la compréhension commune des objectifs de développement national à long terme dans le 
contexte des changements climatiques ainsi qu'un mandat pour mettre le processus clé en adéquation avec cette vision.

(iv) la mise en œuvre décrit la qualité, la quantité et l'orientation stratégique des mesures qui sont appliquées pour améliorer la résilience ou réduire la vulnérabilité aux changements climatiques ;

(v) l'intégration consiste à intégrer l'adaptation aux processus de développement à tous les niveaux de la planification y compris dans les documents et programmes stratégiques nationaux, sectoriels et communaux ;

(vi) la participation fait référence à la participation des représentants des entités privées et publiques ainsi que de la société civile avec une importance toute particulière d'impliquer les représentantes des femmes qui sont souvent touchées de manière disproportionnée par les changements climatiques;

(vii) le suivi- évaluation (S\&E) permet d'identifier les bonnes pratiques et de distinguer les approches moins efficaces, ce qui contribue à une prise de décisions fondée sur des données probantes ainsi qu'à une responsabilisation accrue.

\subsubsection{Sélection des participants à l'atelier de l'évaluation}

Les changements climatiques constituent une dimension transversale qui touche simultanément de nombreux secteurs de développement. C'est pourquoi, l'outil SNAP recommande la participation des acteurs de diverses institutions. Cela a guidé l'identification des parties prenantes à l'atelier d'évaluation participative des capacités et des lacunes pour la planification nationale de l'adaptation. Cet atelier a lieu du 17 au 19 septembre 2019 à Koudougou, dans la région du Centre-Ouest et a regroupé un échantillon de 31 personnes qui reste toutefois supérieur à l'échantillon de 25 participants recommandé par la GIZ.

La majorité des participants $(n=7)$ proviennent des départements du MEEVCC ((Tableau 1).

Les autres acteurs sont des représentants du Ministère de 1'Eau et de l'Assainissement (MEA) $(\mathrm{n}=3)$, du Ministère de l'Agriculture et des Aménagements Hydro-Agricoles (MAAHA) $(n=2)$, du Ministère de l'Energie $(\mathrm{ME})(\mathrm{n}=1)$, du Ministère des Ressources Animales et Halieutiques (MRAH) $(n=2)$, du Ministère en charge de la Femme et de l'Action Humanitaire (MFAH) (n=2), du Ministère des Transports (MT) $(n=2)$, du Ministère de la Santé (MS) $(n=1)$, du Ministère de l'Economie, des Finances et du Développement (MINEFID) $(n=2)$, de la Société Civile $(n=2)$, du Secteur privé $(n=1)$, des projets et programmes $(n=3)$ et des Centres de recherche et Universités $(n=3)$. Il faut également noter que soixante-onze (71) pour cent des participants étaient des hommes et vingt-neuf(29) pour cent des femmes. 
Tableau 1. Parties prenantes à l'évaluation des capacités de planification de l'ACC

\begin{tabular}{|c|c|c|c|}
\hline \multirow[t]{2}{*}{ Institutions } & \multicolumn{3}{|c|}{ Représentants } \\
\hline & Homme & Femme & Total \\
\hline $\begin{array}{l}\text { Ministère en charge de } \\
\text { l'Agriculture } \\
\text { (MAAHA) }\end{array}$ & 2 & 0 & 2 \\
\hline $\begin{array}{c}\text { Ministère des } \\
\text { Ressources Animales } \\
\text { et Halieutiques } \\
\text { (MRAH) }\end{array}$ & 2 & 0 & 2 \\
\hline $\begin{array}{c}\text { Ministère en charge de } \\
\text { l'Environnement } \\
\text { (MEEVCC) }\end{array}$ & 6 & 1 & 7 \\
\hline $\begin{array}{l}\text { Ministère de l'Eau et } \\
\text { de l'Assainissement } \\
\text { (MEA) }\end{array}$ & 2 & 1 & 3 \\
\hline $\begin{array}{l}\text { Ministère de l'Energie } \\
\text { (ME) }\end{array}$ & 1 & 0 & 1 \\
\hline $\begin{array}{l}\text { Ministère en charge de } \\
\text { la Femme et de } \\
\text { l'Action Humanitaire } \\
\text { (MFAH) }\end{array}$ & 0 & 2 & 2 \\
\hline $\begin{array}{c}\text { Ministère des } \\
\text { Transports (MT) }\end{array}$ & 0 & 1 & 1 \\
\hline $\begin{array}{l}\text { Ministère de la Santé } \\
\text { (MS) }\end{array}$ & 0 & 1 & 1 \\
\hline $\begin{array}{l}\text { Ministère en charge de } \\
\text { l'Economie } \\
\text { (MINEFID) }\end{array}$ & 3 & 0 & 3 \\
\hline $\begin{array}{l}\text { Centres de recherche } \\
\text { et Universités }\end{array}$ & 1 & 2 & 3 \\
\hline Secteur Privé & 1 & 0 & 1 \\
\hline Société Civile & 1 & 1 & 2 \\
\hline Projets et programmes & 3 & 0 & 3 \\
\hline Total & 22 & 09 & 31 \\
\hline
\end{tabular}

\subsubsection{Evaluation des capacités nationales de planification de l'ACC}

Les participants ont examiné à l'aide du questionnaire des sept facteurs de réussite de l'outil SNAP, en répondant à trois questions par facteur, pour un total de 21 questions. Pour chaque question, ils ont attribué une note en fonction de l'échelle suivante : $0=$ très faible $; 1=$ faible $; 2=$ moyen ; $3=$ fort et $4=$ très fort. Les notes attribuées ont fait l'objet de justifications, ce qui a permis de formuler des actions pour l'amélioration des capacités actuelles de l'ACC. 


\subsection{Analyse des données}

L'évaluation SNAP a permis d'obtenir des données mixtes (qualitatives et quantitatives) qui ont été saisies dans l'outil SNAP-Excel. L'outil Excel associé au SNAP a permis d'avoir des informations sur l'écarttype qui informe sur le degré de consensus des participants. Ainsi, des valeurs élevées pour l'écart-type soulignent une vision très hétérogène des participants d'une différente compréhension des questions posées. Au contraire, un faible écart-type indique un fort consensus des participants en relation avec la question posée.

\section{Résultats et discussions}

\subsection{Capacités moyennes pour planifier et exécuter effectivement l'adaptation au Burkina Faso}

Les résultats de l'évaluation SNAP des situations actuelle et souhaitée des capacités en matière de planification de l'ACC montrent des variations d'un facteur de succès à l'autre (Tableau 2). Tous les facteurs de réussite du niveau actuel présentent des notes comprises entre 1 et 2 ; ce qui implique que les participants perçoivent que le pays dispose de capacités faibles à modérées pour entreprendre des actions d'adaptation à l'heure actuelle. Le suiviévaluation, la mise en œuvre et la participation ont obtenu les notes les plus faibles soit 1,$6 ; 1,9$ et 1,7 respectivement. Des résultats similaires ont été obtenus au Bénin et au Sénégal avec l'application de l'outil SNAP (GIZ, 2017, 2018). A ce titre, Saito (2013) révèle que les outils climatiques devraient (i) reconnaitre la nécessité de l'intégration, (ii) avoir un dispositif clair de suiviévaluation, (iii) traiter les questions de capacité de mise en œuvre, (iv) impliquer les financiers potentiels dans le processus et (v) associer de manière significative les principales parties prenantes, en particulier les ministères en charge des finances et/ou de la planification.

Ces facteurs de succès de l'intégration ont été également mentionnés par les acteurs nationaux de l'étude. Par ailleurs, cet article montre que les résultats diffèrent de ceux obtenus par Karani et Kariuki (2017), qui ont rapporté qu'au Kenya, la participation du public à la planification et à la prise de décision en matière d'ACC ainsi que la coordination des interventions dans ce domaine avaient les scores moyens les plus élevés. Ils ont également démontré que les acteurs institutionnels avaient des connaissances faibles sur les changements climatiques.

En revanche, le suivi-évaluation de l'adaptation est couramment à la traîne par rapport aux autres composantes de la planification de l'adaptation comme l'atteste de nombreux auteurs (Tang et al., 2010 ; Preston et al., 2011).

En ce qui concerne les objectifs stratégiques, tous les facteurs de réussite à l'exception du suivi-évaluation ont obtenu des notes supérieures ou égales à 3 ; ce qui témoigne une ambition réaliste des acteurs pour l'amélioration 
significative des capacités pour la planification de l'ACC dans les années à venir.

Les facteurs de succès où les participants ont les objectifs stratégiques les plus élevés sont le renforcement de l'intégration dans les outils de planification et l'augmentation de la participation nationale à l'adaptation.

L'évaluation des facteurs de réussite indique des perceptions très peu différentes de la situation actuelle de l'ACC comme le montre l'écart-type généralement faible et compris entre 0,32 et 0,53 .

Il existe également un large consensus sur la nécessité d'une amélioration significative de tous les facteurs de succès en tant qu'objectif stratégique pour le pays, étant donné le faible écart type allant de 0,14 à 0,43.

Tableau 2. Evaluation des capacités et besoins en matière de planification nationale de

l'ACC

\begin{tabular}{ccccc}
\hline Facteurs de succès & \multicolumn{2}{c}{ Niveau actuel } & \multicolumn{2}{c}{ Niveau souhaité } \\
\cline { 2 - 5 } & Moyenne & Ecart type & Moyenne & Ecart type \\
\hline $\begin{array}{c}\text { Information } \\
\text { climatique }\end{array}$ & 2,2 & 0,32 & 3,2 & 0,27 \\
\hline $\begin{array}{c}\text { Capacités } \\
\text { humaine et } \\
\text { institutionnelle }\end{array}$ & 2,4 & 0,44 & 3,2 & 0,27 \\
\hline $\begin{array}{c}\text { Vision à long } \\
\text { terme et mandat }\end{array}$ & 2,5 & 0,35 & 3 & 0,25 \\
\hline Mise en oeuvre & 1,9 & 0,44 & 3 & 0,43 \\
\hline Intégration & 2,3 & 0,53 & 3,6 & 0,29 \\
\hline Participation & 1,7 & 0,51 & 3,4 & 0,21 \\
\hline Suivi et évaluation & 1,6 & 0,34 & 2 & 0,14 \\
\hline
\end{tabular}

\subsection{Principales raisons de l'état actuel des capacités de planification et de mise en ouvre de l'ACC}

\subsubsection{L'information climatique}

La disponibilité des informations climatiques renforce véritablement les capacités d'adaptation des acteurs (Abdul-Razak et Kruse, 2017 ; Devkota et Phuyal, 2018 ; Ayedegue et al., 2020). A l'échelle nationale, les données climatiques sont produites par diverses structures notamment les institutions de recherche, les universités, les projets et l'Agence Nationale de la Météorologie (ANAM). Cependant, les métadonnées sous-régionales du Centre d'Agriculture, d'Hydrologie et de Météorologie (AGRHYMET) et du Comité permanent Inter-Etats de Lutte contre la Sécheresse dans le Sahel (CILSS) sont souvent utilisées comme alternative mais elles ne permettent pas toujours de produire des résultats détaillés fiables.

Les informations climatiques (IC) sont généralement inaccessibles en raison de l'absence de coordination et de partage systématique desdites informations avec les utilisateurs (Vincent et al., 2017, Bremer et al., 2019). Aussi, elles 
sont payantes au niveau de l'ANAM qui est l'institution nationale de production de données climatiques.

Les IC ne couvrent pas tous les secteurs d'activités et les zones du pays. Des études récentes conduites au Burkina Faso (D’haen et Thekritoff, 2019), au Malawi (Vincent et al., 2017), en Caraïbes (Howard, 2018) et au Sénégal (Ouédraogo et al., 2018 ; Sultan et al., 2020) confirment le caractère incomplet des informations existantes sur le climat et les difficultés d'accès des données climatiques scientifiques aux niveaux régional et local.

Les acteurs nationaux ont également relevé des contraintes liées à la collecte, à l'interprétation et à l'utilisation des données sur les changements climatiques (Karani et Kariuki, 2017). Ce constat est corroboré par Lemos et al (2007) qui ont suggéré que l'amélioration de la capacité d'adaptation consiste à investir dans la production et la diffusion ainsi que la communication de l'information. L'insuffisance de moyens financiers, le manque d'expertise pour la modélisation et les capacités limitées à comprendre ou à utiliser les IC constituent les défis majeurs des services climatiques (Sultan et al., 2020).

\subsubsection{Les capacités humaines et institutionnelles}

Le pays dispose d'un PNA qui est assorti de mesures d'ACC. Malheureusement, ce document politique climatique est faiblement diffusé auprès du public et a une faible appropriation par les ministères sectoriels et les autres acteurs. Cette situation serait liée entre autres à la mobilité des ressources humaines et à l'absence d'un mécanisme de coordination intra et intersectorielle. D’une manière générale, les capacités humaines et institutionnelles sont insuffisantes sur la planification de l'ACC dans les ministères et leurs partenaires. Aussi, des divergences sur les connaissances techniques sur l'adaptation existent entre les parties prenantes du PNA dont certains sont plus en avance que d'autres. Ces résultats corroborent les recherches antérieures sur l'importance du leadership, des ressources financières et humaines ainsi que de la communication pour la planification de l'adaptation (Moser et Ekstrom, 2010 ; Hamin et al., 2014).

\subsubsection{La vision à long terme et mandat}

Le PNA mentionne les aspects et l'ampleur ainsi que les effets néfastes des changements climatiques. En effet, il s'appuie sur les informations scientifiques de plusieurs études (GIEC, 2007 ; LAME, 2012). La vision du PNA est que « le Burkina Faso gère plus efficacement son développement économique et social grâce à la mise en auvre de mécanismes de planification et de mesures prenant en compte la résilience et l'adaptation aux changements climatiques à l'horizon 2050 ». Les principaux outils de planification (i) le Plan National de Développement Economique et Social (PNDES) et (ii) le deuxième Programme National du Secteur Rural (PNSR) sont en cohérence 
avec le PNA. Dans ces documents nationaux, les changements climatiques sont présentés comme étant une barrière au développement du pays et l'ACC est mentionnée comme étant une option obligatoire et incontournable (D'haen et Thekritoff, 2019). Les participants ont également relevé des barrières institutionnelles à la réalisation du facteur de vision et du mandat qui sont l'absence de mécanisme de coordination du PNA et l'insuffisance de ressources humaines et financières. A cela s'ajoute, le manque de dispositifs législatifs favorables à la prise en compte de l'ACC dans les documents politiques de planification. D'autres auteurs corroborent que la multiplicité des acteurs impliqués dans la prise de décision en matière d'adaptation avec l'insuffisance de coordination entraîne des mandats peu clairs, des actions contradictoires et la fragmentation des efforts d'adaptation (Biesbroek et al., 2011 ; Scobie, 2016).

\subsubsection{La mise en ouvre}

Les participants considèrent que des efforts sont faits en matière de cohérence entre les actions d'adaptation et les priorités nationales. Cet alignement avec les priorités nationales est un critère fondamental de sélection des bailleurs de fonds internationaux pour l'octroi des aides financières. Cependant, la mise en œuvre des politiques, des stratégies et des plans est confrontée aux faiblesses des ressources financières et de l'expertise technique (Spires et al., 2014 ; Robinson et Doran, 2017 ; Ouattara et al., 2019). Aussi, il n'existe pas de budgétisation explicite de l'adaptation aux niveau national, sectoriel et local. Cela rejoint les conclusions de l'étude de Karani et Kariuki (2017) qui ont indiqué que le Kenya n'avait pas encore alloué de financements aux actions climatiques.

L'Accord de Paris sur le Climat de 2015 reconnait également que les fonds publics ne seront pas suffisants pour lutter contre les changements climatiques. À cet égard, le Burkina Faso doit garantir un environnement favorable pour les initiatives du Secteur Privé et de la Société civile. Par ailleurs, l'accréditation du Fonds d'Intervention pour l'Environnement (FIE) et de Coris Bank International (CBI) au Fonds Vert pour le Climat constitue un atout pour le financement des actions d'ACC à travers le pays.

\subsubsection{L'intégration}

L'intégration est de plus en plus une réalité en ce sens que certaines politiques nationales d'adaptation et de développement au Burkina Faso prennent en compte les mesures d'ACC (D'haen et Thekritoff, 2019). Malgré l'existence au niveau national de guides méthodologiques d'intégration des changements climatiques dans la planification, les politiques nationales et les plans communaux de développement (PCD) ainsi que les plans régionaux de développement (PRD) ne prennent pas suffisamment en compte les mesures 
d'ACC. Cela pourrait être lié à l'absence d'une masse critique d'experts dans le domaine des changements climatiques et la faible appropriation des outils climatiques nationaux par les acteurs clés du processus de planification. Audelà de ces lacunes, les participants ont souligné les faibles possibilités de prise de décision au niveau décentralisé et la faible collaboration d'une part entre les institutions étatiques et d'autre part entre les structures non étatiques et étatiques. Plusieurs auteurs ont également rapporté que l'absence de l'implication des décideurs aux niveaux local, régional et national est l'une des limites actuelles de l'intégration des informations climatiques dans les outils de planification (Rose, 2014 ; Vincent et al., 2017 ; Ouédraogo et al., 2018). L'actualisation des PCD et l'élaboration des PRD constituent des points d'entrée privilégiés pour l'intégration de l'adaptation aux niveaux régional et local. Aussi, le futur référentiel national de développement, consécutif au PNDES, offre l'opportunité de mieux prendre en compte l'ACC dans le processus national de planification et de budgétisation.

\subsubsection{La participation}

Le processus d'élaboration du PNA du pays a impliqué la participation de plusieurs acteurs nationaux.

Cependant, ce processus a connu une faible consultation des populations à la base et une faible implication de certains acteurs des secteurs privé et financier. Cela pourrait être lié à la difficulté d'identification des groupes vulnérables du processus PNA qui ne participent pas tous de manière significative audit processus.

Le PNA est peu connu au niveau local et n'est pas pris en compte dans les PCD et les PRD. Or, la meilleure façon d'aborder l'adaptation est de le faire au niveau local ou régional car les conditions et les impacts climatiques varient au niveau de ces deux échelles (Measham et al., 2011 ; Termeer et al., 2011; Nalau et al., 2015). Les acteurs nationaux trouvent également que l'insuffisance de ressources financières et le manque d'autonomisation des groupes vulnérables sont de véritables contraintes à leur pleine participation.

\subsubsection{Le suivi-évaluation}

L'Accord de Paris demande aux Parties à la CCNUCC de partager les informations sur les progrès réalisés en matière d'adaptation. Nonobstant cette disposition, le Burkina Faso n'a pas encore élaboré de système national de S\&E dédié aux changements climatiques. Cependant, l'expertise en matière de S\&E des projets/programmes et des actions de développement est disponible dans les ministères sectoriels et les collectivités territoriales. D'une manière générale, ces mécanismes nationaux de $S \& E$ n'intègrent pas suffisamment les indicateurs spécifiques aux changements climatiques et au genre. Cela s'explique par le fait que les indicateurs au niveau du PNA 
manquent de précisions en termes de calcul, de valeurs de référence et de ciblage. A cela s'ajoute, l'insuffisance de collaboration entre les acteurs et l'insuffisance des ressources humaines et financières. Des résultats similaires ont été observés par Howard (2018) qui estime que la moitié des planificateurs (47\%) ne participe pas au S\&E de l'adaptation et que leurs institutions respectives ne l'envisagent pas actuellement.

L'évaluation des politiques publiques reste relativement faible au Burkina Faso avec des confusions dans la compréhension des concepts de suivi et de contrôle, souvent assimilés à l'évaluation (Sombié, 2019). Cela est en partie imputable à l'absence d'un cadre légal et règlementaire régissant la pratique de l'évaluation et à la faiblesse des capacités évaluatives nationales.

Plusieurs auteurs (UNFCCC, 2010 ; Spearman et McGray, 2011) soutiennent que le $\mathrm{S} \& \mathrm{E}$ de l'adaptation est important pour la responsabilisation des acteurs et l'évaluation des performances des initiatives d'adaptation. Il est également reconnu la nécessité de modifier les approches conventionnelles de S\&E pour répondre aux besoins de l'ACC (Olivier et al., 2012).

\subsection{Principales actions d'amélioration de la planification de l'ACC}

Au cours de l'atelier d'évaluation des capacités nationales de l'ACC, les facteurs de succès avec les plus faibles notes concernent le suiviévaluation, la capacité de mise en œuvre et le niveau de participation. Plusieurs actions ont été formulées par les parties prenantes pour l'amélioration du niveau actuel des capacités de planification de l'adaptation. Ces actions intègrent les sept facteurs de succès et se regroupent en quatre catégories :

- Premièrement, la mobilisation des ressources nécessaires pour la planification de l'ACC en général et plus particulièrement la mise en œuvre du PNA. Le manque de ressources se manifeste dans les étapes de production des information climatiques, de mise en œuvre, de participation et d'intégration ainsi que de suivi-évaluation de la planification de l'adaptation. A cet effet, des ressources financières doivent être allouées aux mesures d'adaptation, ce qui nécessite véritablement la levée de fonds nationaux et internationaux. Au-delà de la mobilisation des financements, il s'avère indispensable de renforcer les capacités institutionnelles et humaines à tous les niveaux pour la prise en compte des changements climatiques dans les documents de planification stratégiques ;

- Deuxièmement, l'intégration de l'ACC dans la planification nationale, sectorielle et locale, pour permettre une intégration réussie de l'adaptation. Les acteurs pensent que la fonction de coordination générale doit être consacrée à un organe central pourvu d'un pouvoir décisionnel par rapport aux ministères c'est-à-dire la Primature ou le Ministère chargé de l'économie et de la planification. L'intégration de l'adaptation dans la planification s'appuie sur la production des informations sur la variabilité 
du climat. A cet effet, le pays doit œuvrer à la production de ses propres données climatiques et renforcer les études de vulnérabilité. Il doit également améliorer la disponibilité de données sur le climat et les projections climatiques à tous les utilisateurs potentiels. La participation des acteurs non-gouvernementaux peut aussi contribuer à l'application de mesures d'adaptation à différents niveaux ;

- Troisièmement, la mise en place d'un dispositif de S\&E fonctionnel et sensible au genre qui permette de mesurer les efforts enregistrés. Le pays a opté de s'appuyer véritablement sur les systèmes de S\&E existants pour l'intégration du S\&E de l'adaptation et de l'atténuation aux changements climatiques. Cette voie semble pragmatique et économique pour la collecte des données à travers les plateformes intersectorielles déjà établies. Toutefois, il y a la nécessité d'harmoniser les systèmes nationaux de S\&E y compris leurs indicateurs, les types de données entre les différentes échelles, les secteurs et les institutions pour permettre d'établir des comparaisons. Les acteurs pourraient utiliser le catalogue national d'indicateurs de développement élaboré depuis 2010 pour la planification et le suivi-évaluation des politiques, des projets et des programmes. Ce catalogue d'indicateurs est promu par l'Observatoire National de l'Environnement et du Développement Durable (ONEDD).

Pour garantir la fonctionnalité de ce mécanisme de S\&E, il est indispensable de renforcer les capacités opérationnelles des différents acteurs notamment la Cellule de S\&E au sein du SP-CNDD, les Points focaux des Ministères sectoriels et les collectivités territoriales ainsi que la Société civile. Ce renforcement de capacités devrait être orienté sur (i) la connaissance et l'utilisation de l'information climatique, (ii) les aspects clés des PNA, (iii) les stratégies de mobilisation de la finance climatique et (iv) le système de suiviévaluation;

- Quatrièmement, l'amélioration de l'implication des parties prenantes en donnant plus de place aux organisations de femmes et en renforçant le dialogue multi-acteurs aux niveaux national et local. La collaboration entre les organes nationaux, locaux et sectoriels est nécessaire pour la mise en œuvre effective du PNA et sa bonne appropriation par toutes les parties prenantes. Ainsi, il a été proposé d'inclure les acteurs des secteurs privé et bancaire dans l'élaboration, la mise en œuvre et le suivi-évaluation des actions relatives à la planification de l'adaptation. Il faut également assurer une coordination unifiée de l'ensemble des Partenaires Techniques et Financiers (PTF) intervenant dans le domaine de l'adaptation.

L'ACC requiert la participation de toutes les parties prenantes et le partenariat reste une contribution essentielle pour la mise en œuvre effective des actions identifiées par les acteurs. L'importance du partenariat a été également signalée par Somda et al. (2014) lors de l'analyse participative de la 
vulnérabilité et de la planification de l'ACC dans le Nord du Burkina Faso. Les auteurs suggèrent que les initiatives d'adaptation doivent s'appuyer sur le principe d'actions et de partenariat.

\subsection{Leçons apprises de l'évaluation des capacités nationales de planification de l'ACC}

L'application de l'outil SNAP a fourni une base de référence sur les capacités nationales en matière de planification de l'ACC. De ce processus, quatre principaux enseignements ont été identifiés notamment (i) la disponibilité des ressources humaines et financières, (ii) la vulgarisation des résultats de l'évaluation auprès des parties prenantes, (iii) l'approche participative et inclusive et (iv) la reproduction et l'extension de l'évaluation SNAP.

\subsubsection{Adapter l'évaluation à la disponibilité des ressources humaines et financières}

L'évaluation des capacités en matière d'adaptation est exigeante en termes de capacités humaines et de ressources financières. Il faut donc une coordination forte qui implique un niveau élevé des acteurs de prise de décisions. L'échantillon des participants de la présente étude a été défini en lien avec les limites du budget disponible. Cependant, l'étude suggère que le questionnaire de l'outil SNAP soit envoyé aux acteurs nationaux si des contraintes budgétaires existent aux fins de disposer d'une taille d'échantillon plus importante. Cette alternative permettra à chaque participant au préalable de se documenter et d'apporter des réponses précises et objectives.

\subsubsection{Vulgariser les résultats de l'évaluation auprès des parties prenantes}

Plusieurs acteurs institutionnels jouent un rôle important dans la réussite du processus PNA. Pour améliorer les buts stratégiques de l'adaptation, l'évaluation a identifié une panoplie d'actions avec des structures responsables de leur mise en œuvre.

A ce titre, il est indispensable de partager les conclusions de l'évaluation avec ces parties concernées pour clarifier les différentes responsabilités et influencer le processus de planification de l'ACC. Au-delà de ces acteurs, le grand public pourrait être informé des résultats présentés dans un rapport non technique et synthétique. L'analyse des capacités nationales pour la planification de l'ACC a permis d'identifier les besoins et les lacunes en matière d'adaptation. Cette approche a fourni des informations utiles pour la prise de décision dans la lutte contre les changements climatiques. 


\subsubsection{Utiliser l'approche participative et inclusive}

L'approche de l'outil SNAP est fondée sur l'implication effective des principales parties prenantes du processus de planification et de suiviévaluation du PNA. Cette approche utilisée a suscité beaucoup d'engouement et d'engagement de la part des participants qui ont identifié de façon consensuelle les principales barrières à la planification des mesures d'adaptation et les recommandations y afférentes. Les acteurs ont vu leur capacité technique renforcée à travers les différentes communications développées au cours de l'atelier et axées sur les informations climatiques et les expériences des projets sur l'intégration des changements climatiques dans la planification. Ils sont également en mesure d'évaluer l'état des lieux des capacités de planification de l'ACC au niveau national.

\subsubsection{Reproduire et étendre l'évaluation SNAP}

L'adaptation est littéralement complexe et les progrès en la matière peuvent s'observer sur les moyen et long termes. La reproduction de l'évaluation SNAP sur deux périodes différentes serait bénéfique pour appréhender véritablement l'évolution de la capacité des autorités nationales et locales dans l'opérationnalisation des activités déclinées dans les outils climatiques de l'ACC. Toutefois, la mise en œuvre de ce cadre méthodologique implique de formuler de manière plus précise les questions pour permettre une meilleure notation objective, de définir des indicateurs pour l'évaluation des différents facteurs de réussite du PNA et d'impliquer toujours les mêmes participants pour le processus d'évaluation. L'application de l'outil SNAP au niveau sectoriel pourrait être associée à des outils d'analyse de vulnérabilité aux changements climatiques pour faciliter la sensibilisation et rendre résilients les investissements dans le secteur concerné. En effet, la sensibilisation sociale et politique sur les changements climatiques permet d'avoir le soutien à l'adaptation du public et des autres parties prenantes à tous les niveaux de la société (Biesbroek et al., 2011). En revanche, l'évaluation de la vulnérabilité aux changements climatiques constitue une étape essentielle de la planification de l'adaptation. En outre, elle permet d'une part d'établir les liens existants entre le climat et le développement et d'autre part d'améliorer la compréhension et le suivi de l'évolution possible de certains risques climatiques dans diverses zones géographiques. En ce sens, Mastrandrea et al. (2010) soutiennent que l'amélioration du lien entre la vulnérabilité du climat et les décisions publiques et privées en matière de planification est l'un des défis essentiels de l'ACC.

\section{Conclusion}

La planification de l'ACC est considérée comme un outil clé pour faire progresser l'action sur la réduction de la vulnérabilité aux impacts climatiques. 
Dans la présente étude, l'outil SNAP basé sur les facteurs de réussite du processus PNA, a été utilisé pour évaluer les capacités nationales de l'ACC au Burkina Faso. Les résultats montrent que les forces du processus PNA sont la disponibilité de l'information climatique et des capacités humaines et institutionnelles, l'existence d'un PNA, l'intégration relative de l'ACC dans les outils de planification. En revanche, les faibles capacités du processus du PNA sont (i) l'insuffisance de la mise en œuvre des mesures d'adaptation dans certains secteurs, (ii) la faible implication des acteurs autour de l'ACC et (iii) l'absence de mécanisme de suivi-évaluation des mesures d'adaptation issus du PNA. Ces faiblesses impliquent que l'accent doit être mis sur le soutien à l'amélioration de la capacité des organisations individuelles et du pays à planifier et à mettre en œuvre des actions d'adaptation. Le pays dispose de potentialités pour améliorer sa capacité de planification de l'ACC. Les différentes leçons identifiées peuvent constituer des éléments de base pour la révision du PNA du pays : (i) adapter la disponibilité des ressources humaines et financières, (ii) vulgariser les résultats de l'évaluation auprès des parties prenantes, (iii) adopter l'approche participative et inclusive et (iv) reproduire et étendre l'évaluation SNAP.

\section{References :}

1. Abdul-Razak, M., \& Kruse, S. (2017) The Adaptive Capacity of Smallholder Far-mers to Climate Change in the Northern Region of Ghana. Climate Risk Management, 17: 104-122.

2. Adger, W., Huq, S., Brown, K., Conway, D., \& Hulme, M. (2003). Adaptation to climate change in the developing world. Progress in Development Studies, 3:179-195.

3. Ayedegue, L.U., Issaka, K., \& Yabi, A.J. (2020). Typologie et déterminants des stratégies d'adaptation aux changements climatiques en riziculture au nord et centre du Bénin. European Scientific Journal, ESJ, 16 (6), 206-234.

4. Banque Mondiale, (2017). Amélioration de la connaissance et de la gestion des eaux au Burkina Faso, s.1.: s.n.

5. Barbir,J., Leal Filho, W., \& Spiric, J. (2009). An appraisal of measures to cope with climate change in the Baltic Sea. Management of Environmental Quality, 20 :82-91.

6. Biesbroek, R., Klostermann, J., Termeer, C., \& Kabat, P. (2011). Barriers to climate change adaptation in the Netherlands. Climate law, 2(2): 181-199.

7. Bremer, S., Wardekker, A., Dessai, S., Sobolowski, S., Slaattelid, R., \& van der Sluijs, J. (2019). Toward a multi-faceted conception of coproduction of climate services. Climate Services, 13:42-50. 
8. Chapin, F.S., Kofinas, G.P., \& Folke, C. (2009) Principles of ecosystem stewardship: resilience based natural resource management in a changing world. Springer, London.

9. D'haen, S., \&Thekritoff, E. (2019). État des lieux de l'intégration du changement climatique dans les politiques nationales d'adaptation et de développement au Burkina Faso. 53 p. Report produced under the project 'Projet d'Appui Scientifique aux processus de Plans Nationaux d'Adaptation dans les pays francophones les moins avancés d'Afrique subsaharienne", Climate Analytics gGmbH, Berlin.

10. Dayamba, S. D., D’haen, S., Coulibaly, O. J. D., \& Korahiré, J. A. (2019). Étude de la vulnérabilité des systèmes de production agrosylvo-pastoraux face aux changements climatiques dans les provinces du Houet et du Tuy au Burkina Faso.49p. Report produced under the project 'Projet d'Appui Scientifique aux processus de Plans Nationaux d'Adaptation dans les pays francophones les moins avancés d'Afrique subsaharienne", Climate Analytics gGmbH, Berlin.

11. Devkota, N., \& Phuyal, R.K. (2018) Adoption Practice of Climate Change Adaptation Options among Nepalese Rice Farmers: Role of Information and Communication Technologies (ICTs). American Journal of Climate Change, 7, 135-152.

12. Diarra, A., Barbier, B., Zongo, B., \& Yacouba, H. (2017). Impact of climate change on cotton production in Burkina Faso. African Journal of Agricultural Research. Vol. 12(7), pp. 494-501.

13. Gadédjisso-Tossou, A., Egbendewe, A. Y. G., \& Abbey, G. A. (2016). Assessing the impact of climate change on smallholder farmers' Crop Net Revenue in Togo. Journal of Agriculture and Environment for International Development, 110 (2): 229-248.

14. GIEC. (2014). Changements climatiques 2014: incidences, adaptation et vulnérabilité- Résumé à l'intention des décideurs. Contribution du Groupe de travail II au cinquième Rapport d'évaluation du Groupe d'experts intergouvernemental sur l'évolution du climat Organisation Météorologique Mondiale, Genève (Suisse), 34

15. GIEC. (2007). Bilan 2007 des changements climatiques. Contribution des Groupes de travail I, II et III au quatrième Rapport d'évaluation du Groupe d'experts intergouvernemental sur l'évolution du climat [Équipe de rédaction principale, Pachauri, R.K. et Reisinger, A. (publié sous la direction de)]. GIEC, Genève, Suisse, 103p.

16. GIZ. (2018). Etat lieux des capacités de planification aux changements climatiques au Sénégal. 36 p. Report produced under the project "Projet d'Appui Scientifique aux processus de Plans Nationaux d'Adaptation dans les pays francophones les moins avancés d'Afrique subsaharienne". 
17. GIZ. (2017). Etat des lieux des capacités pour la planification de l'adaptation aux changements climatiques au Bénin. 59 p. Report produced under the project "Projet d'Appui Scientifique aux processus de Plans Nationaux d'Adaptation dans les pays francophones les moins avancés d'Afrique subsaharienne".

18. GIZ. (2014). L'outil diagnostic de l'état des lieux pour la planification de l'adaptation au niveau national (SNAP). $4 \mathrm{p}$. https://www.adaptationcommunity.net/?wpfb_dl=149

19. Hamin, E. M., Gurran, N., \& Emlinger, A. M. (2014). Barriers to Municipal Climate Adaptation: Examples From Coastal Massachusetts' Smaller Cities and Towns. Journal of the American Planning Association, 80(2): 110-122.

20. Howard, D. (2018). Exploring the Linkages Between Planning and the Barriers to Climate Change Adaptation in Caribbean Small Island Developing States. PHD, University of Waterloo, Canada, 328 p.

21. Hurlimann, A.C., \& March, A.P. (2012) The role of spatial planning in adapting to climate change. Wiley Interdisciplinary ReviewsClimate Change, 3:477-488.

22. Ibrahim, B., Polcher, J.; Karambiri, H., Yacouba, H., \& Ribstein, P. (2014). Changes in rainfall regime over Burkina Faso under the climate change conditions simulated by 5 regional climate models. Climate Dynamics, 42 : 1363-1381.

23. INSD (Institut National de la Statistique et de la Démographie). (2017).

Annuairestatistique2016.370p.http://www.insd.bf/n/contenu/pub_peri odiques/annuaires_stat/Annuaires_stat_nationaux_BF/Annuaire_stat_ 2016.pdf

24. Karani, I., \& Kariuki, N. (2017). Using Participatory Approaches in Measuring Resilience and Development in Isiolo County, Kenya. J.I. Uitto et al. (eds.), Evaluating Climate Change Action for Sustainable Development.

25. LAME (Laboratoire d'Analyses Mathématiques des Equations). (2012). Elaboration du PANA Programmatique du Burkina Faso : Etudes de modélisation climatique, d'évaluation des risques et analyse de la vulnérabilité aux changements climatiques. Les projections de climat futur au Burkina Faso. LAME, Unité de Formation et de Recherche en Sciences Exactes et Appliquées, Université de Ouagadougou.

26. Lejeune, Q., \& Saeed, F. (2019). Étude de l'impact des changements climatiques futurs sur les ressources en eau au Burkina Faso. Report produced under the PAS-PNA project. Climate Analytics $\mathrm{GmbH}$, Berlin. 29 p. 
27. Lemos, M. C., Boyd, E., Tompkins, E.L., Osbahr, H., \& Liverman, D. (2007). Developing adaptation and adapting development. Ecology and Society, 12(2): 26.

28. 28.Mastrandrea, M.D., Heller, N.E., Root, T.L., \& Schneider, S.H. (2010). Bridging the gap: linking climate-impacts research with adaptation planning and management. Climatic Change, 100: 87-101.

29. Measham, T., Preston, B., Smith, T., Brooke, C., Gorddard, R., Withycombe, G., \& Morrison, C. (2011). Adapting to climate change through local municipal planning: barriers and challenges. Mitigation and Adaptation Strategies for Global Change, 16:889-909.

30. MERH (Ministère de l'Environnement et des Ressources Halieutiques), (2015). Plan National d'Adaptation aux changements climatiques du Burkina Faso. 155 p.

31. Millner, A., \& Dietz, S. (2015). Adaptation to climate change and economic growth in developing countries. Environment and Development Economics, 20 (03): 380-406.

32. Moser, S. C., \& Ekstrom, J. A. (2010). A framework to diagnose barriers to climate change adaptation. Proceedings of the National Academy of Sciences, 107(51): 22026-22031.

33. Nalau, J., Preston, B.L., \& Maloney, M.C. (2015) Is adaptation a local responsibility? Environmental Science \& Policy, 48: 89-98.

34. N'drin, O., J. A., Konan-Waidhet, A.B., \& Kienon-Kabore, T.H. (2019). Analyse des déterminants de la résilience aux changements climatiques des cultivateurs du département de Fresco, Côte d'Ivoire. European Scientific Journal, ESJ, 15 (18), 288-314.

35. Niang, I., Ruppel, O.C., Abdrabo, M.A., Essel, A., Lennard, C., Padgham, J., \& Urquhart, P. (2014). Africa, Climate Change 2014: Impacts, Adaptation and Vulnerability - Contributions of the Working Group II to the Fifth Assessment Report of the Intergovernmental Panel on Climate Change., p 1199-1265.

36. Olivier, J., Leiter, T., \& Linke, J. (2012). Adaptation made to measure: A guidebook to the design and results-based monitoring of climate change adaptation projects, Manual. Deutsche Gesellschaft für Internationale Zusammenarbeit (GIZ). www.seachangecop.org/node/1661.

37. Ouattara, I., Diarra, Y., \& Mariko, S. (2019). Etude des impacts des changements climatiques sur les activités agricoles dans la Commune Rurale de Mafouné, Cercle de Tominian, Région de Ségou au Mali. European Scientific Journal, ESJ, 15 (11), 121-144.

38. Ouedraogo, I., Diouf, N.S., Ouédraogo, M., Ndiaye, O., \& Zougmoré, R.B. (2018). Closing the Gap between Climate Information Producers 
and Users: Assessment of Needs and Uptake in Senegal. Climate, 6 : 13.

39. Panthou, G., Lebel, T., Vischel, T., Quantin, G., Sane, Y., Ba, A., Ndiaye, O., Diongue-Niang, A., \& Diopkane, M. (2018). Rainfall intensification in tropical semi-arid regions: the Sahelian case. Environmental Research Letters, 13 (2018) 064013.

40. Pielke, Sr., Adegoke, J., BeltraáN-Przekurat, A., Hiemstra, C.A., Lin, J., Nair, U. S., Niyogi, D., \& Nobis.T. E. (2007) An overview of regional land-use and land-cover impacts on rainfall, Tellus B: Chemical and Physical Meteorology, 59 (3): 587-601.

41. Pramova, E., Locatelli, B., Brockhaus, M., \& Fohlmeister, S. (2012). Ecosystem services in the national adaptation programmes of action. Climate Policy, 12(4): 393-409.

42. Preston, B. L., Westaway, R. M., \& Yuen, E. J. (2011). Climate adaptation planning in practice: An evaluation of adaptation plans from three developed nations. Mitigation and Adaptation Strategies for Global Change (Vol. 16).

43. Robinson, S. A., \& Dornan, M. (2017). International financing for climate change adaptation in small island developing states. Regional Environmental Change, 17(4): 1103-1115.

44. Rose, D.C. (2014). Five ways to enhance the impact of climate science. Nature Climate Change, 4, 522.

45. Saito, N. (2013). Mainstreaming climate change adaptation in least developed countries in South and Southeast Asia. Mitigation and Adaptation Strategies for Global Change, 18:825-849.

46. Salack, S., Sarr, B., Sangare, S. K., Ly, M., Sanda, I. S., \& Kunstmann, H. (2015). Crop-Climate Ensemble Scenarios To Improve Risk Assessment And Resilience In The Semi-Arid Regions Of West Pays En Afrique. Climate Research, 65: 107-121.

47. Scobie, M. (2016). Policy coherence in climate governance in Caribbean small island developing states. Environmental Science \& Policy, 58: 16-28.

48. Sombié, I. (2019). Système de suivi-évaluation des actions climatiques au Burkina Faso. Report produced under the project 'Projet d'Appui Scientifique aux processus de Plans Nationaux d'Adaptation dans les pays francophones les moins avancés d'Afrique subsaharienne", Deutsche Gesellschaft für Internationale Zusammenarbeit (GIZ), Berlin, $77 \mathrm{p}$.

49. Somda, J., Sawadogo, I., Savadogo, M., Zougmoré, R., Bationo, B. A., Moussa, A. S., Nakoulma, G., Sanou, J., Barry, S., Sanou, A. O., \& Some, L. (2014). Participatory vulnerability assessment and planning of adaptation to climate change in the Yatenga, Burkina Faso. CGIAR 
Challenge Program on Climate Change, Agriculture and Food Security is available on line at www.ccafs.cgiar.org, $44 \mathrm{p}$.

50. Spearman, M., \& McGray, H. (2011). Making adaptation count: Concepts and options for monitoring and evaluation of climate change adaptation. Eschborn: Gesellschaft für Internationale Zusammenarbeit (GIZ) GmbH. //pdf.wri.org/making_adap tation_count.pdf

51. Spires, M., Shackleton, S., \& Cundill, G. (2014). Barriers to implementing planned community based adaptation in developing countries: a systematic literature review. Climate and Development, 6(3): 277-288.

52. Sultan, B., Lejeuneb, Q., Menkeb, I., Maskellb,G., Leeb, K., Nobletb, M., Syc, I., \& Roudierd, P.( 2020). Current needs for climate services in West Africa: Results from two stakeholder surveys. Climate Services, 18 (2020) 100166.

53. Tang, Z., Brody, S. D., Quinn, C., Chang, L., \& Wei, T. (2010). Moving from agenda to action: evaluating local climate change action plans. Journal of Environmental Planning and Management, 53(1): $41-62$.

54. Termeer, C., Dewulf, A., van Rijswick, H., van Buuren, A., Huitema, D., Meijerink, S., Rayner, T., \& Wiering, M. (2011). The regional governance of climate adaptation: a framework for developing legitimate, effective, and resilient governance arrangements. Climate Law, 2: 159-179.

55. Traore, S., \& Owiyo, T. (2013). Dirty droughts causing loss and damage in Northern Burkina Faso. International Journal of Global Warming, 5 (4), 2013.

56. UNFCCC (United Nations Framework Convention on Climate Change). (2010). National adaptation programmes of action (NAPA). Available http://unfccc.int/national_reports/napa/items/2719.php

57. Vincent, K., Dougill, A.J., Dixon, J.L., Stringer, L.C., \& Cull, T. (2017). Identifying climate services needs for national planning: Insights from Malawi. Climate Policy, 17: 189-202. 